\title{
Juno at Jupiter
}

David Stevenson

Citation: Physics Today 73, 9, 62 (2020); doi: 10.1063/PT.3.4577

View online: https://doi.org/10.1063/PT.3.4577

View Table of Contents: https://physicstoday.scitation.org/toc/pto/73/9

Published by the American Institute of Physics

\section{ARTICLES YOU MAY BE INTERESTED IN}

An Alaskan volcano, climate change, and the history of ancient Rome

Physics Today 73, 17 (2020); https://doi.org/10.1063/PT.3.4563

Graphene gets bent

Physics Today 73, 46 (2020); https://doi.org/10.1063/PT.3.4569

Dawn of the topological age?

Physics Today 73, 30 (2020); https://doi.org/10.1063/PT.3.4567

Does new physics lurk inside living matter?

Physics Today 73, 34 (2020); https://doi.org/10.1063/PT.3.4546

Could hydrogen bail out nuclear power?

Physics Today 73, 20 (2020); https://doi.org/10.1063/PT.3.4543

Cats and llamas could offer a path to coronavirus therapies

Physics Today 73, 22 (2020); https://doi.org/10.1063/PT.3.4565

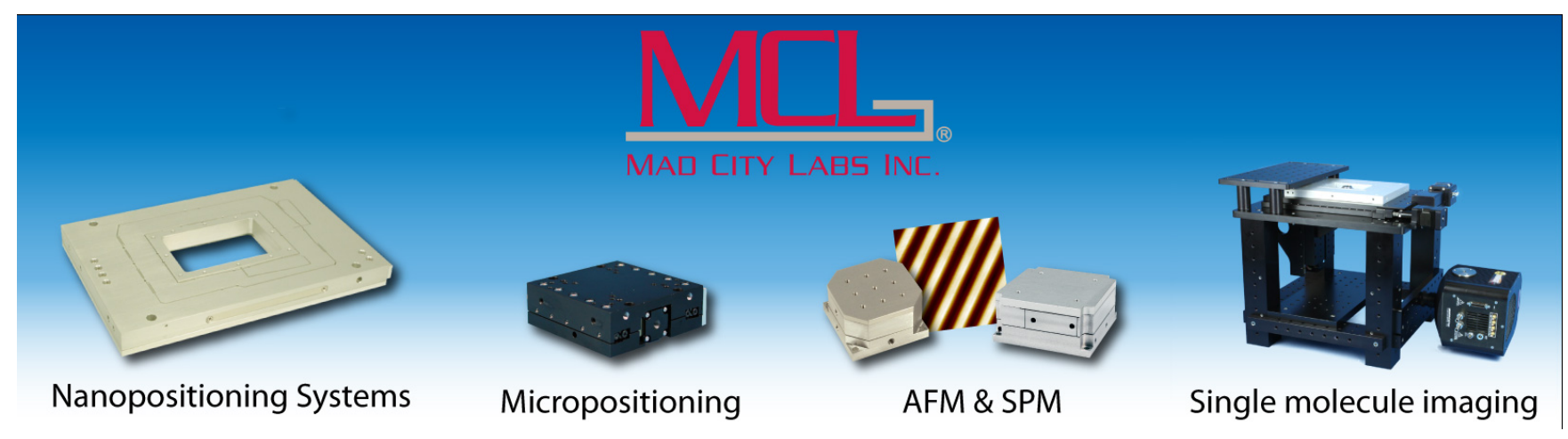




\section{Juno at Jupiter}

\section{David Stevenson}

\section{A NASA spacecraft in orbit around Jupiter is yielding new and surprising information about our solar system's dominant planet.}

upiter is in the class of planets known as gas giants - those planets primarily made from hydrogen and helium gas, which, under gravitational compression, become metallic fluids. It's the most massive planet in our solar system - so massive that it influences everything else, including the delivery of material to Earth. Jupiter is also thought to have formed first among the planets, perhaps a few million years after a cloud of gas and dust from the interstellar medium collapsed and led to our solar system 4.6 billion years ago. That influence means that we need to understand the planet's composition and structure to understand how the solar system came into being.

Through its program of deep space missions,

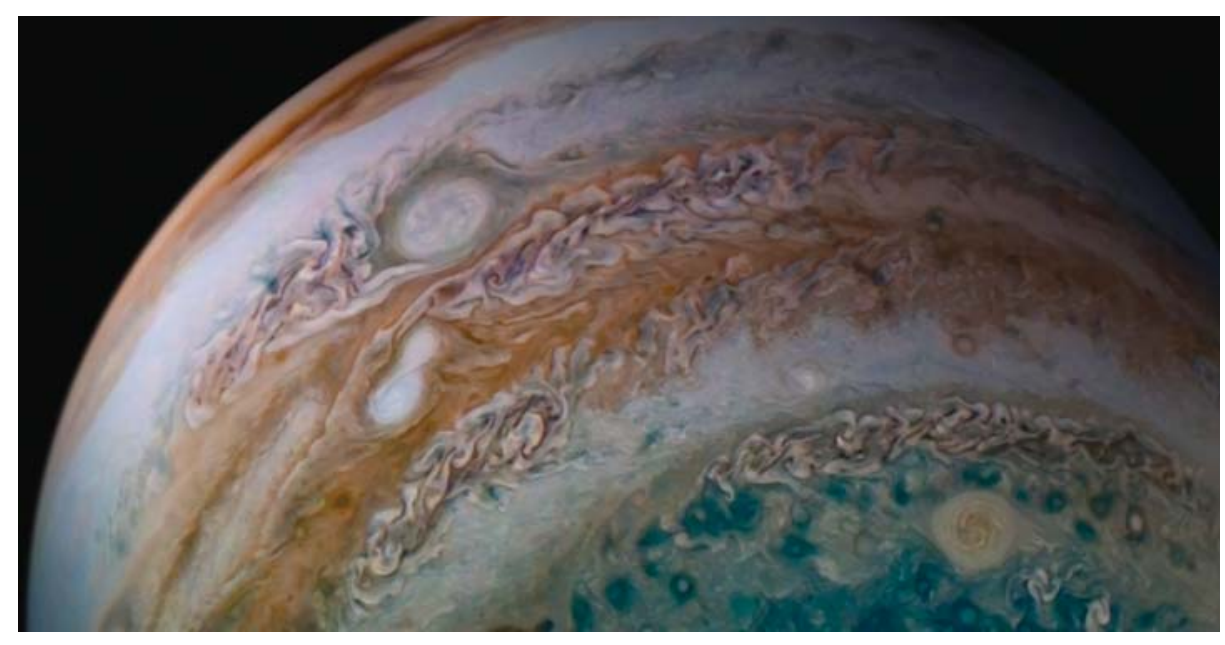

NASA has long recognized the importance of Jupiter. But the planet remains mysterious. Does it have a core? What is the nature of its magnetic field? How do the deep atmosphere and strong winds behave? How much water does it have? To answer those questions, one must visit the planet. For that job, a billion-dollar New Frontiers mission spacecraft named Juno was chosen in 2003, launched in 2011, and went into Jovian orbit on 4 July 2016. The mission, led by Scott Bolton of the Southwest Research Institute, is still collecting data. But it's already made some important measurements. This Quick Study outlines three types: The first has to do with gravity; the second, with the magnetic field; and the third, with passive radiation from inside the planet.

\section{Gravity: high harmonics and deep winds}

When a spacecraft orbits a planet, its velocity is determined primarily by the gravitational potential it experiences. If Jupiter were spherically symmetric, then Juno's orbit would be explained by Kepler's laws and we would only learn about the planet's mass - assuming we know Newton's constant $G$ from lab experiments, not an easy task. But Jupiter is not spherically symmetric because it rotates, and the rotation is not uniform with latitude and depth.

Jupiter is oblate, a feature that shows up as a gravitational quadrupole and causes a precession in Juno's elliptical orbit. The winds also express themselves in Juno's trajectory. The spacecraft's tracking ability, enabled by a microwave link to NASA's Deep Space Network, is so exquisitely good-better than a factor of 1 part in 10 million in the gravity potentialthat scientists can determine higher-order harmonics of the gravity field and discern differences between northern and southern hemispheres caused by the differences in the winds

FIGURE1. THIS VIEW OF JUPITER'S SWIRLING ATMOSPHERE features two storms caught in the act of merging. The two white ovals, left of center in the orange-colored band, are anticyclonic storms that rotate counterclockwise. (Image courtesy of NASA/JPLCaltech/SwRI/MSSS; image processing by Tanya Oleksuik.)

in those regions. The gravity harmonics, together with interior modeling of the planet, have allowed researchers to infer much about Jupiter's internal structure.

The simplest interpretation of the higher harmonics is that the planet has a diluted central concentration of heavy elements rather than a compact central core of them. The difference amounts to a mere 10 to 15 Earth masses, a small fraction of the planet's total mass. Even so, current ideas of Jupiter formation favor the accumulation of a planetary embryo from that tiny mass before the mostly hydrogen envelope accumulated on top. The dilution of those heavy elements and hydrogen is not easily explained. But that interpretation is the first of several surprises Juno has provided. One possible explanation is a giant impact during Jupiter's formation, an event that might have stirred up a primordial core.

Before Juno, some researchers claimed that the winds in Jupiter's atmosphere are purely meteorological-that is, confined to a thin layer extending a mere $100 \mathrm{~km}$ in depth. Others argued they are a surface expression of convective flows that extend as much as 20000 to $30000 \mathrm{~km}$ deep, limited only by the region where hydrogen gas becomes liquid metal. The truth appears to be somewhere in the middle. Jupiter has strong winds (see figure 1) that extend perhaps $3000 \mathrm{~km}$ deep, judging by the harmonics of the gravity field (see PHYSICS TODAY, May 2018, page 19). Below that depth, the winds taper off because of the unavoidable electromotive force that arises from Fara- 

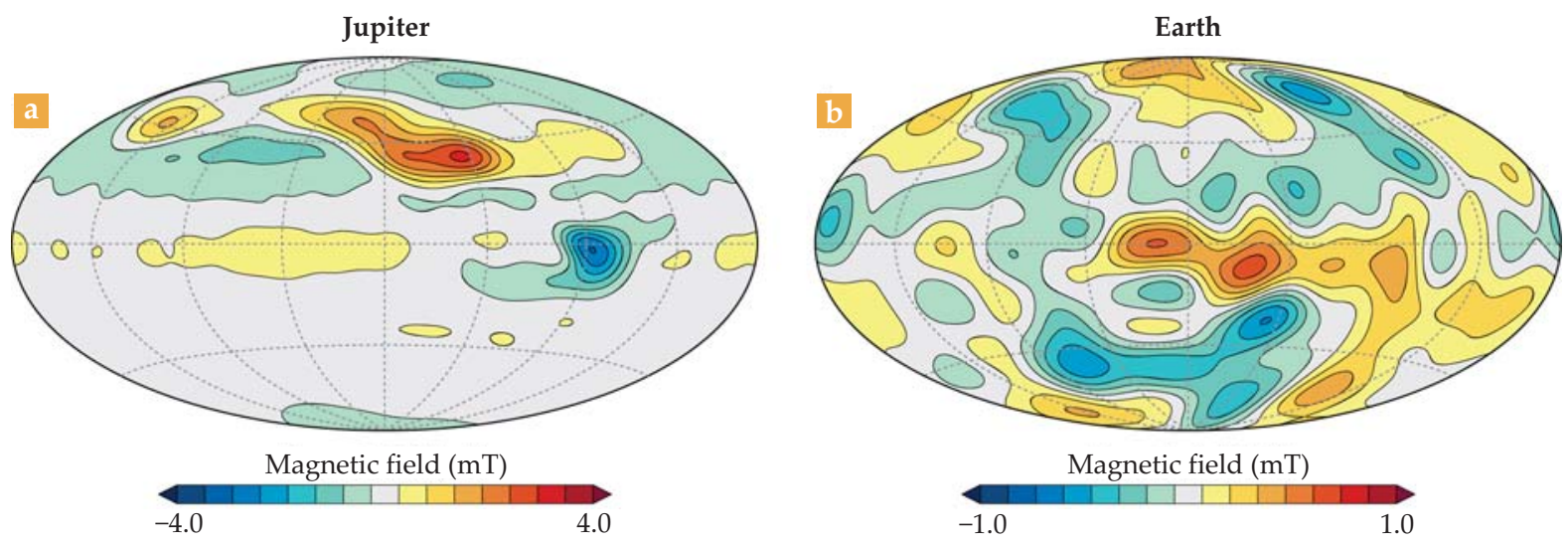

FIGURE 2. JUPITER'S AND EARTH'S MAGNETIC FIELDS. (a) Almost all of Jupiter's nondipole radial field, simulated here at 0.9 of Jupiter's radius, is concentrated in the northern hemisphere. (b) Earth's nondipole field, by contrast, is evenly distributed throughout. (Adapted from D. J. Stevenson, Annu. Rev. Earth Planet. Sci. 48, 465, 2020.)

day's law when the electrically conducting wind flows across magnetic field lines.

The consequences of the flows aren't well understood, but we know that for typical winds and field strengths, an electrical conductivity similar to that of salty water or the human body, roughly 1 siemens per meter, leads to a substantial change in field strength. That conductivity is orders of magnitude less than that of a typical metal and occurs at about $97 \%$ of Jupiter's radius, well outside the region where the main magnetic field is produced but at a place where the winds appear to decline. Still, planetary scientists lack a dynamical explanation for how the winds decline with depth. The nature of the winds on Jupiter - and those on Saturn, Uranus, Neptune, and exoplanets - will likely remain a lively topic of future research, stimulated by Juno's discoveries.

\section{Magnetic field: northern exposure}

Scientists have been aware of Jupiter's large magnetic field since the early days of radio astronomy in the 1950s, and previous spacecraft have documented a field that was somewhat Earthlike (with a similar dipole tilt and similar ratio of quadrupole to dipole) after taking into account a field-generating region that extends closer to the surface than Earth's. However, Juno has discovered some unexpected features in the magnetic field, whose spatial distribution is strongly heterogeneous (see figure 2). The planet exhibits sparse but strong fields confined to small regions, most notably the planet's Great Blue Spot-a region of downward flux near the equator.

Learning about magnetic fields in our solar system has been a sobering experience. It seems that whenever we measure anew we find something different, and Jupiter is no exception. Like Earth, however, Jupiter has an internal magnetic field that changes in time. The cause is presumably the advection of magnetic field lines by flow within the planet (see the article by Peter Olson, PHYSICS TODAY, November 2013, page 30). In Jupiter's case, though, the advection could be telling us about the connection between the field and the winds. That connection would suggest an interaction between magnetic and gravity fields.

\section{Microwave radiation: a watery mystery}

I've saved the most remarkable discovery for last. It is common practice in the planetary community to think of clouds in a convective atmosphere as being the surface expression of a well- mixed region beneath them, with the base of the cloud deck defined simply by equating the partial pressure of the cloudforming material to its local vapor pressure. In that picture, ammonia should appear as well mixed within Jupiter deeper than a pressure of about one bar-the surface pressure of Earth.

The microwave radiometer on Juno picks up the planet's interior radiation, which is attenuated by intervening ammonia. Astonishingly, though, it did not see that well-mixed state. Instead, it generally saw a factor-of-two depletion in ammonia, except for a narrow band near the equator. If ammonia is not well mixed, what are we to say about water, which, like ammonia, is a condensable species? Water abundance in Jupiter is one of the Juno mission's key goals. Assuming the narrow band is also well mixed in water, Juno's microwave data suggest that water is at least as abundant as it would be if the atmosphere had the same elemental abundances as the Sun. And it might be two or three times more abundant.

Still, we are confronted with the conclusion that what is happening in most of Jupiter's atmosphere is poorly understood. The mystery is confounding for interior models as well because they depend on knowing the atmospheric boundary condition. Lots of ammonia resides at the top of the atmosphere and we expect a lot in the interior, but at issue is how to transport it. The best way to hide a lot of transported ammonia from observation is to deliver it quickly but in a spatially localized way. One proposed solution invokes a form of hail known as mushballs, capable of rapidly delivering ammonia and water to great depths without evaporation. Perhaps future observations can help us unravel that unsettling result.

Juno is ongoing; it has not answered all questions and has raised new ones - a measure of success. Plans are afoot to extend the mission, with more discoveries promised and even a possible trip to the Galilean moons.

\section{Additional resources}

- S. J. Bolton et al., "Jupiter's interior and deep atmosphere: The initial pole-to-pole passes with the Juno spacecraft," Science 356, 821 (2017).

- S. J. Bolton et al., "The Juno mission," Space Sci. Rev. 213, 5 (2017).

- D. J. Stevenson, "Jupiter's interior as revealed by Juno," Annu. Rev. Earth Planet. Sci. 48, 465 (2020). 